\title{
Restrict Antibiotic Usage among Healthy Animals to Prevent the Spread of Antimicrobial Resistance
}

\section{Saurabh RamBihariLal Shrivastava* and Prateek Saurabh Shrivastava}

Department of Community Medicine, Shri Sathya Sai Medical College and Research Institute, Tamil Nadu, India

The threat of Antimicrobial Resistance (AMR) has raised serious concerns on the advances made in the field of prevention and treatment of numerous infections resulting because of different pathogenic organisms [1]. Owing to the emergence of the AMR, the outcome of any major surgical procedure or chemotherapy for malignancies/common infections has become questionable and the time is not far, when people will start losing their lives due to the earlier treatable infections $[1,2]$. A wide range of factors like indiscriminate and irrational use of antibiotics, use of antibiotics among food-producing animals, etc., has been attributed in the causation of AMR [1,2].

The factor of extensive use of antibiotics among food-producing animals has resulted in the development of antimicrobial-resistant bacteria, which can be then transmitted from animals to humans either through direct contact or via the food chain [3]. Such resulting infections are usually chronic, augment the incidence of hospitalization and even aggravate the chances of treatment failure [4]. It is extremely important to know that more than 0.4 million people die due to food-borne diseases and if the causative organisms (like Salmonella, Escherichia coli, etc.) become resistant, huge number of people will be dying because of the same [3].

In order to contain the problem in the agriculture sector, the World Health Organization has released a set of recommendations [3]. These recommendations call for an overall decrease in the use of all classes of medically important antimicrobials in food-producing animals and total restriction of the same among the food-producing animals for the purpose of growth promotion or for prevention of those infections that have not been diagnosed [4]. These recommendations should be strictly adhered as they remain the best way to avert the risk of the transmission of antimicrobial-resistant bacteria from food-producing animals to humans $[3,4]$.

Moreover, the findings of a systematic review indicated that by implementation of measures to restrict the use of antibiotics among food-producing animals, a decline of close to $40 \%$ was observed in the incidence of AMR [3]. Further, it has been advocated to use only those antibiotics among animals that are minimally useful for human health [3]. In addition, it has been envisaged to test the animals to ascertain the most effective antibiotic, if possible [2,3]. Also, there is a simultaneous need to improve animal hygiene, promote better use of vaccines and modify animal housing and husbandry practices [2-4].

To conclude, the new recommendations have been framed to maintain the effectiveness of antibiotics that are important for use among humans. Further, owing to the availability of very few drugs in the research pipeline, there is an immense need to mount a strong and sustained response to promote the rational use of antibiotics among animals.

\section{References}

1. Shrivastava SR, Shrivastava PS, Ramasamy J (2015) Much more is expected from nations to counter antimicrobial resistance: World Health Organization. J Res Med Sci 20: 719-720.

2. World Health Organization (2017) Antimicrobial resistance in the food chain.

3. World Health Organization (2017) Stop using antibiotics in healthy animals to prevent the spread of antibiotic resistance.

4. World Health Organization (2017) WHO guidelines on use of medically important antimicrobials in food-producing animals.
*Corresponding author: Saurabh RamBihariLal Shrivastava, Department of Community Medicine, Shri Sathya Sai Medical College and Research Institute, Tamil Nadu, India, Tel: 919884227224; Email: drshrishri2008@gmail.com

Received: October 16, 2018; Accepted: October 23, 2018; Published: October 30, 2018

Citation: Shrivastava SR, Shrivastava PS (2018) Restrict Antibiotic Usage among Healthy Animals to Prevent the Spread of Antimicrobial Resistance. Prim Health Care 8: e126. doi: 10.4172/2167-1079.1000e126

Copyright: (C) 2018 Shrivastava SR, et al. This is an open-access article distributed under the terms of the Creative Commons Attribution License, which permits unrestricted use, distribution, and reproduction in any medium, provided the original author and source are credited. 